\title{
Safety of Intradermal Rabies Vaccination as Pre Exposure Prophylaxis
}

Ravish Hardanahalli S*, Jayanthi Srikanth, Rachana Annadani, Pradeep Kumar DP, Malatesh Undi, Chandana Krishna, Rupsa Banerjee, Vairava Solai and Aravind Manoharan

Department of Community Medicine, Kempegowda Institute of Medical Sciences (KIMS), Bangalore, India

*Corresponding author: Ravish Hardanahalli Shankaraiah, Associate Professor, Department of Community Medicine, Kempegowda Institute of Medical Sciences (KIMS), Banashankari $2^{\text {nd }}$ Stage, Bangalore, India, Tel: +919900562743; E-mail: drravishhs@rediffmail.com

Received date: November 17, 2016; Accepted date: December 19, 2016; Published date: January 02, 2017

Copyright: (c) 2016 Shankaraiah $\mathrm{RH}$, et al. This is an open-access article distributed under the terms of the Creative Commons Attribution License, which permits unrestricted use, distribution, and reproduction in any medium, provided the original author and source are credited.

\begin{abstract}
:
Need for the study: WHO recommends pre exposure prophylaxis in rabies endemic countries for all individuals who are at increased risk of exposure to rabies such as veterinarians, rag-pickers, animal handlers, rabies researchers/laboratory workers, International travellers and children.
\end{abstract}

Objectives: To assess the safety of rabies vaccine administered intradermally as pre-exposure prophylaxis among 3 high risk groups viz., children, rag-pickers and veterinarians.

Methods: All the subjects who volunteered from each of the 3 risk groups were taken for the study and were given purified chick embryo cell rabies vaccine having a potency of $>2.5 \mathrm{IU}$ per dose, intradermally as pre exposure prophylaxis on days 0,7 and 21 . Subsequently, all were followed up to assess the safety of intradermal rabies vaccination by recording the adverse drug reactions from the day of administration of first dose of vaccination upto 2 weeks after the last dose of vaccination.

Results: The present study included 150 children, 225 rag-pickers and 122 veterinary students. The adverse drug reactions to rabies vaccination among these groups were $5.1 \%, 5.0 \%$ and $10.4 \%$ respectively which were mild in nature and subsided spontaneously or by taking symptomatic treatment and none of the subjects dropped out because of adverse drug reactions.

Conclusion: Pre exposure prophylaxis against rabies by intradermal route is safe and well tolerated by high risk groups, which may be considered as a strategy for eliminating the disease by 2030 .

Keywords: Safety; Pre exposure prophylaxis; Intradermal rabies vaccine; Children; Veterinary students; Rag-pickers

\section{Introduction}

Human rabies is a viral zoonotic disease that occurs in over 150 countries and territories covering all the continents of the World, except Antarctica. The disease that is practically $100 \%$ fatal, poses a potential threat to over 3.3 billion people worldwide with dog as the main transmitter of the disease [1]. In this regard, world health organization (WHO) in a recent meeting at Geneva in December, 2015 has called for elimination of dog-mediated human rabies from the world by 2030 [2].

Rabies in humans is preventable with the help of WHO recommended immunobiologicals either as pre-exposure prophylaxis (PrEP) or post-exposure prophylaxis (PEP). WHO recommends pre exposure prophylaxis for all individuals who are at increased risk of exposure to rabies, because of their residence or occupation; such as veterinarians, rag-pickers, animal handlers, rabies researchers/ laboratory workers, International travellers and children [3]. Preexposure vaccination consists of three doses of vaccine given in deltoid region on days 0,7 and 21/28. It is safe, cost effective and has several advantages; as it simplifies management after exposure to animal bites in future, by eliminating the need for rabies immunoglobulin (RIG) which is expensive/not available at all time/place and also decreases the number of doses of vaccine to only 2 on day 0 and 3 . It may also provide partial immunity to persons who seek delayed post-exposure prophylaxis and might provide some protection to persons at risk of unrecognized exposures to rabies.

In developing countries, where the cost of intramuscular rabies vaccination is the major limiting factor, intradermal rabies vaccination is a safe and immunogenic alternative which reduces the volume and direct cost of vaccine. Therefore, WHO has recommended intradermal use of rabies vaccine as pre- and post-exposure prophylaxis since 1991 [4].

Pre exposure vaccination may be considered for all the children living in endemic countries especially in urban poor areas, as they are at higher risk for exposure, since a combination of large human and dog populations in congested habitable areas combined with widespread poverty may lead to more exposures. Similarly, they play outdoors, hence vulnerable to dog bites as the unvaccinated stray dogs are frequently present on the streets/public places and school playgrounds and these small children are unable to protect themselves when they are attacked by dogs. Moreover, children may not report the minor bites/delay the reporting to their parents, because of fear.

Similarly, rag-pickers in developing countries, which collect thrash daily on the streets, will invariably have continuous contact with stray animals and are at high risk of exposure. Likewise, veterinary students, 
Page 2 of 3

who will continuously handle all the animals in rabies endemic countries as a part of their academics, are a high risk group.

In this background, the study was conducted to assess the safety of rabies vaccine administered intra-dermally as pre-exposure prophylaxis among the 3 high risk groups viz., children and rag-pickers of urban poor locality and veterinarians.

\section{Materials and Methods}

The study was conducted after getting clearance from Institutional Ethics Committee. The present study included 3 groups of subjects:

Group 1: Children from an urban poor locality.

Group 2: Rag-pickers

Group 3: Government Veterinary college students

This study was done as a part of World Rabies Day Activity 2015 from Kempegowda Institute of Medical Sciences (KIMS), Bangalore. At the outset, all the study subjects were given health education regarding prevention of rabies and importance of pre-exposure rabies vaccination for their high risk nature.

Group 1: A publicity campaign was arranged regarding free preexposure rabies vaccination for children in an urban poor locality. Later, the vaccination was done on fixed days at the urban health training centre of KIMS, Bangalore. All the children who volunteered for the vaccination were included in the study and the informed consent from their parents who accompanied them and assent from the study subjects was taken.

Group 2: A publicity campaign was arranged regarding free preexposure rabies vaccination for all rag-pickers in their government colony at the outskirts of Bangalore city. Later, the vaccination was done on fixed days at Government School. All the Rag pickers who volunteered for the vaccination were included in the study and the informed consent from the study subjects was taken.

Group 3: Prior permission was obtained from the Dean of the veterinary college for conducting the study. Later, the vaccination was done on fixed days at the Government Veterinary College, Bangalore. All the Students who volunteered for the vaccination were included in the study and the informed consent from the study subjects was taken.

The socio demographic profile of all the study subjects of the 3 groups was recorded. Subsequently, all of them were given $3(0.1 \mathrm{ml})$ intradermal pre-exposure rabies vaccination on days 0,7 and 21 in the deltoid area by the trained doctors. The vaccine administered was purified chick embryo cell rabies vaccine having a potency of $>2.5$ IU per single dose.

After each of the vaccination dose, the subjects were observed for an hour, to find out any adverse drug reactions (ADRs) and then, a follow-up card was given to enter any ADRs during the entire period of vaccination and two weeks after the last dose. All the reported ADRs were recorded and analysed.

At the end of the study, all the vaccines were given pre-exposure prophylaxis certification as a document. The assessment of rabies virus neutralising antibodies after 1 year in randomly selected study subjects has been planned as a follow-up to know the immunogenicity of pre exposure prophylaxis after 1 year.

\section{Results}

\section{Socio-demographic profile}

Group 1: 150 children of urban poor locality in the age group of 5-10 years with the mean age 7.29 years were included in the study. Among them 90 were boys and 60 were girls. All the children belonged to either class IV/V socio economic status.

Group 2: 225 rag-pickers in the age group of 18-60 years with the mean age 27.9 years were included in the study. Among them 160 were males and 65 were females. All the study subjects belonged to below poverty line.

Group 3: 122 Government veterinary college students in the age group of 18-25 years with the mean age 19.26 years were included in the study. Among them 62 were males and 60 were females.

\section{Safety and tolerability}

Group 1: 150 children received 3 doses of pre exposure rabies vaccination intradermally, totalling to 450 doses of vaccine. In the study subjects, 23 (5.1\%) adverse drug reactions were reported among which 18 were local and 5 were systemic reactions. The solicited local reactions were pain at the injection site $14(3.1 \%)$, redness $2(0.4 \%)$ and injection site itching $2(0.4 \%)$ which subsided without any medication. The systemic ADRs being fever in $3(0.6 \%)$ and myalgia in $2(0.4 \%)$, All reactions were mild and subsided without any complications. None of the study subjects dropped out due to adverse reactions.

Group 2: 225 rag-pickers took 3 doses of vaccine, accounting to 675 doses. Among them, 34 (5.1\%) adverse reactions were reported from 20 subjects. 31 were local reactions and 3 were systemic reactions. All the reactions were mild and subsided without any complications. The solicited local adverse reaction were pain at the injection site $20(3.1 \%)$, redness $2(0.3 \%)$, itching at the site of injection 9 (1.4\%) which subsided without any medication. The systemic ADRs being fever in 1 $(0.2 \%)$ and myalgia in $2(0.3 \%)$, who took basic medications such as anti-inflammatory and were relieved of their symptoms. None of the study subjects dropped out due to adverse reactions (Table 1).

\begin{tabular}{|l|l|l|l|}
\hline $\begin{array}{l}\text { Adverse } \\
\text { reactions }\end{array}$ & $\begin{array}{l}\text { Group } \\
\text { Children }\end{array}$ & $\begin{array}{l}\text { Group 2: Rag- } \\
\text { pickers }\end{array}$ & $\begin{array}{l}\text { Group } \\
\text { Veterinary } \\
\text { students }\end{array}$ \\
\hline Local ADRs & $14(3.1 \%)$ & $20(3.1 \%)$ & $7(2.1 \%)$ \\
\hline Pain & $2(0.4 \%)$ & $2(0.3 \%)$ & $13(3.6 \%)$ \\
\hline Redness & $2(0.4 \%)$ & $9(1.4 \%)$ & $11(3.1 \%)$ \\
\hline Itching & $3(0.6 \%)$ & $1(0.2 \%)$ & - \\
\hline Systemic ADRs & $2(0.4 \%)$ & $2(0.3 \%)$ & - \\
\hline Fever & - & - & $1(0.2 \%)$ \\
\hline Myalgia & - & - & $6(1.6 \%)$ \\
\hline Headache & $23(5.1 \%)$ & $34(5.0 \%)$ & $38(10.4 \%)$ \\
\hline Fatigue & & &
\end{tabular}

Table 1: Adverse reactions following pre-exposure prophylaxis. 
Group 3: 122 veterinary students took 3 doses of intradermal vaccine, accounting to 366 doses. There were 38 (10.3\%) adverse reactions which included, pain at injection site $7(2.1 \%)$, redness 13 (3.6\%), itching at the site of injection $11(3.1 \%)$, fatigue $6(1.6 \%)$ and headache $1(0.2 \%)$. All the reactions were mild and subsided without any complications. None of the study subjects dropped out due to adverse reactions.

\section{Discussion}

Rabies, a vaccine preventable disease, still continues to pose a significant public health problem in many developing countries. Preexposure prophylaxis against rabies is a cost effective tool to prevent the disease and is recommended for anyone who will be at continual, frequent or increased risk of exposure to the rabies virus in canine rabies infected areas. The immunological memory after pre exposure vaccination has been demonstrated to have a long lasting immunity against rabies in humans. It has been shown that, the person who had received vaccination 5-21 years back had good anamnestic response after booster vaccination [5]. Long-term immunity was also seen with intradermal rabies vaccination. Therefore, the anamnestic response to a booster vaccination was not related to the route of administration (intramuscular/intradermal) or to a completed pre-exposure or postexposure vaccination [6].

Pre exposure vaccination has been shown to be safe and effective in children and co-administration of rabies vaccine along with DPT and inactivated polio vaccine at 2 and 4 months and 1 year respectively, elicited satisfactory antibody titres to all antigens with no interference with each other antigens used [7].

The present study showed that intradermal administration of antirabies vaccine as pre-exposure prophylaxis is safe and well tolerated. Similarly, a multi centric study on pre-exposure intradermal rabies vaccination with Purified Chick Embryo Cell Vaccine (PCECV) was found to be safe and immunogenic with production of adequate antibody titers by day 14 and was maintained till the yearly booster doses [8].

Another study conducted among children with PCECV administered as PrEP through intradermal route have proved to be safe and well tolerated with only 25 ADRs for 405 ID doses. None of the study subjects dropped out due to adverse reactions and there were no complications reported [9].

Another study from Thailand demonstrated the safety and immunogenicity of intradermal rabies pre-exposure prophylaxis with purified chick embryo cell vaccine (PCECV) in school children age 5 to 8 years and concluded that rabies pre-exposure immunization with PCECV is safe and immunogenic [10].

In conclusion, the intradermal administration of rabies vaccination as pre-exposure prophylaxis, among high risk groups was found to be safe and well tolerated. This may be considered in the rabies endemic regions as it will save the lives of vulnerable population.

\section{Conflict of Interest}

None

\section{Financial Disclosure}

None

\section{References}

1. WHO position paper (2010) Rabies vaccines. World Health Organization. Weekly Epidemiological Record, 85:309-20.

2. Global Elimination of dog-mediated human rabies.

3. WHO (2013) Expert Consultation on Rabies. Technical Report Series (TRS) 982. World Health Organization, Geneva.

4. World Health Organization (2005) WHO expert consultation on rabies. TRS 931. WHO Geneva.

5. Suwansrinon $\mathrm{K}$, Wilde $\mathrm{H}$, Benjavongkulchai $\mathrm{M}$, Banjongkasaena $\mathrm{U}$, Lertjarutorn S, et al. (2006) Survival of neutralizing antibody in previously rabies vaccinated subjects: a prospective study showing long lasting immunity. Vaccine 24: 3878-3880.

6. Gherardin AW, Scrimgeour DJ, Lau SC, Phillips MA, Kass RB (2001) Early rabies antibody response to intramuscular booster in previously intra dermally immunized travelers using human diploid cell rabies vaccine. J Travel Med, 8: 122-26.

7. Lang J, Duong QH, Nguyen VG, Le TT, Nguyen CV, et al. (1997) Randomised feasibility trial of pre-exposure rabies vaccination with DTPIPV in infants. The Lancet 349: 1663-1665.

8. Malerczyk C, Vakil HB, Bender W, (2013) Rabies pre-exposure vaccination of children with purified chick embryo cell vaccine (PCECV). Hum Vaccin Immunother 9:1454-1459.

9. Haradanahalli SR, Jayanthi S, Narayana DHA, Annadani R, Vijayashankar V, et al. (2013) Pre-exposure prophylaxis against rabies in children. Hum Vaccin Immunother 9: 1910-1913.

10. Thavatchai K, Wirawan T, Phana P, Phran P, Pakamatz K, et.al. (2007) Pre-Exposure Rabies Vaccination Using Purified Chick Embryo Cell Rabies Vaccine Intradermally is Immunogenic and Safe. Journl Paed 151: 173-177. 